This item was submitted to Loughborough's Research Repository by the author.

Items in Figshare are protected by copyright, with all rights reserved, unless otherwise indicated.

\title{
Influence of the voltage waveform's shape and on-time duration on the dissolved ozone produced by a DBD bubble reactor
}

PLEASE CITE THE PUBLISHED VERSION

https://doi.org/10.1088/1361-6595/ab024f

\section{PUBLISHER}

(C) IOP Publishing

\section{VERSION}

AM (Accepted Manuscript)

\section{PUBLISHER STATEMENT}

This is the Accepted Manuscript version of an article accepted for publication in Plasma Sources Science and Technology. IOP Publishing Ltd is not responsible for any errors or omissions in this version of the manuscript or any version derived from it. The Version of Record is available online at https://doi.org/10.1088/13616595/ab024f.

\section{LICENCE}

CC BY-NC-ND 4.0

\section{REPOSITORY RECORD}

Seri, Paolo, Alexander R.P. Wright, Alex Shaw, Felipe Iza, Hemaka Bandulasena, Carlo A. Borghi, and Gabriele Neretti. 2019. "Influence of the Voltage Waveform's Shape and On-time Duration on the Dissolved Ozone Produced by a DBD Bubble Reactor". figshare. https://hdl.handle.net/2134/36783. 


\title{
1 Influence of the voltage waveform's shape and on-time duration on the dissolved
}

\section{ozone produced by a DBD bubble reactor}

\author{
Paolo Seri ${ }^{1,2}$, Alexander Wright ${ }^{2,3}$, Alex Shaw ${ }^{2}$, Felipe Iza $^{2}$, Hemaka Bandulasena $^{3}$, Carlo A Borghi ${ }^{1}$, \\ Gabriele Neretti ${ }^{1 *}$
}

${ }^{1}$ Department of Electrical, Electronic and Information Engineering, University of Bologna 40136, Italy

${ }^{2}$ Wolfson School of Mechanical, Electrical and Manufacturing Engineering, Loughborough University, Loughborough, Leicestershire, LE11 3TU, United Kingdom

${ }^{3}$ Department of Chemical Engineering, Loughborough University, Loughborough, Leicestershire, LE11 3TU, United Kingdom

In this study we examine both the effect of changing the applied voltage waveform shape and the modulation on-time on the amount of ozone dissolved within a liquid in a DBD bubble reactor. In this device, the discharge forms at the gas liquid interface allowing for effective transfer of the plasma effluent into the liquid. To produce different voltage waveforms, a multilevel inverter power supply capable of generating arbitrary waveforms without switching-on and switching-off transients has been used. Of the four waveforms used in the study (sinusoidal, sawtooth, square and short-pulse), the square waveform was found to be the most efficient at producing the highest ozone concentration for a fixed peak voltage and average power. To determine the effect of the modulation on-time, the number of cycles during the on-time were increased from 1 up to 1000 , adjusting the off-time accordingly to maintain the same duty cycle. Shorter on-time periods were found to be more efficient. Experimental and computational results indicate that the time between subsequent discharges is critical for increased ozone generation efficacy, as this needs to be long enough for ozone produced in one discharge event to diffuse away from the discharge region before the next discharge event occurs, thereby avoiding its partial destruction in the plasma. This insight provides a valuable criterion for the optimization of DBD reactors used in novel biomedical, agricultural and environmental applications. 
2 The use of ozone in water treatment is now well established across Europe and North America [1]. Ozone's

3 oxidation potential is superior to that of chlorine and it is effective at inactivating bacteria, removing odors,

4 controlling color and causing flocculation of particles to reduce water turbidity [2]. Most civil applications

5 require large volumes of ozone and large generators have been developed over the years. Dielectric barrier

6 discharges (DBD) are the preferred type of ozone generators [3] and due to the high energy requirement and

7 the need for high efficacy, many operational parameters such as input gas mixture, feed gas temperature,

8 discharge geometry, and electrode size and shape have been investigated [4,5]. A key element for the

9 efficient production of ozone is the waveform with which the power is applied [6,7]. This is typically

10 sinusoidal with frequencies ranging from 10 to $100 \mathrm{kHz}$, with the frequency being a key factor in ozone

11 production too [8-10]. Higher frequencies allow for higher power density and thus ozone concentrations.

12 However, it cannot be assumed that this leads to higher efficiency as several studies point towards improved

13 efficiency at lower frequencies. The optimal frequency is typically system and process dependent [9-11].

Improvements in the production of ozone can be studied via modulation of the plasma. By changing the plasma on-time and/or the duty-cycle, ozone concentrations can be enhanced [12]. Power modulation allows time for the system to cool, thereby limiting the temperature build up in the generator whilst reducing the power consumption in the plasma [12,13]. For example, Olszewski et al. found that at a fixed 50\% dutycycle, an on-time of $10 \mathrm{~ms}$ was more efficient than an on-time of $60 \mathrm{~s}$ as this limited the temperature rise in the device [14]. However, the results presented are rather inconclusive as a shorter on-time of $1 \mathrm{~ms}$ was found to be slightly less efficient than the $10 \mathrm{~ms}$ on-time. Other studies have found that an on-time of $0.5 \mathrm{~ms}$ was more efficient than a $2.8 \mathrm{~ms}$ on-time, highlighting the need for a better understanding of the processes that determine the optimum waveform [15].

There are limited studies related to the influence of the shape of the applied voltage waveform on the production of ozone [16,17], although unipolar waveforms have been found to be less efficient than bipolar waveform in asymmetric DBDs, a difference attributed to charge accumulation on the electrode barrier [6]. In large scale ozone generators, sinusoidal waveforms are commonly used; however, there is limited literature to demonstrate that they are the most effective. 
1 This study explores the effect of waveform shape and plasma on-time duration on the ozone concentration

2 transferred into a liquid in a DBD plasma micro-bubble reactor. Besides direct ozone measurements in the

3 liquid, the degradation of potassium indigo trisulfonate was investigated as a proxy for a water treatment

4 process. Four waveforms are considered in the study, namely sinusoidal, square, pulse and positive sawtooth;

5 and the plasma on-time is varied between $8 \mu$ s to $50 \mathrm{~ms}$. The peak voltage was kept constant for all

6 waveforms and the duty-cycle was adjusted to maintain the average power delivered to the plasma the same

7 in all cases.

\subsection{Materials and methods}

\subsection{Multi-level power supply}

10 The power supply used in this study is high-voltage high-frequency single-phase arbitrary waveform voltage 11 generator, capable of driving a wide range of capacitively loads $[18,19]$. The power supply has a cascade of 1224 H-bridge modules that can deliver 49 output voltage levels (Figure 1). Each individual H-bridge module 13 has a $600 \mathrm{~V}$ DC supply that is powered by a flyback converter fed from a $12 \mathrm{~V}$ DC battery (Figure $1 \mathrm{~b}$ ). A 14 maximum output voltage of $\pm 14.4 \mathrm{kV}(600 \mathrm{~V} x 24)$ can be delivered by the power supply. In this study, only 1514 modules were used because the highest voltage required was less than $8.4 \mathrm{kV}$. An example of each of the four waveforms used in this study (i.e. sinusoidal, square, pulse, and positive sawtooth) are shown in Figure 2.
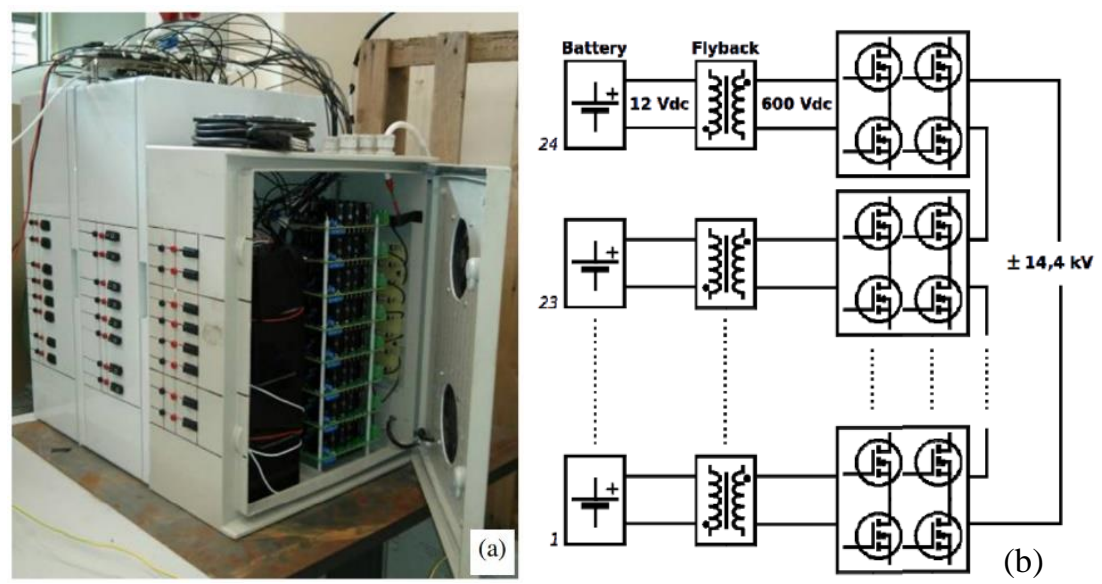

Figure 1 - (a) Multilevel power supply and (b) its electrical topology [19]. 

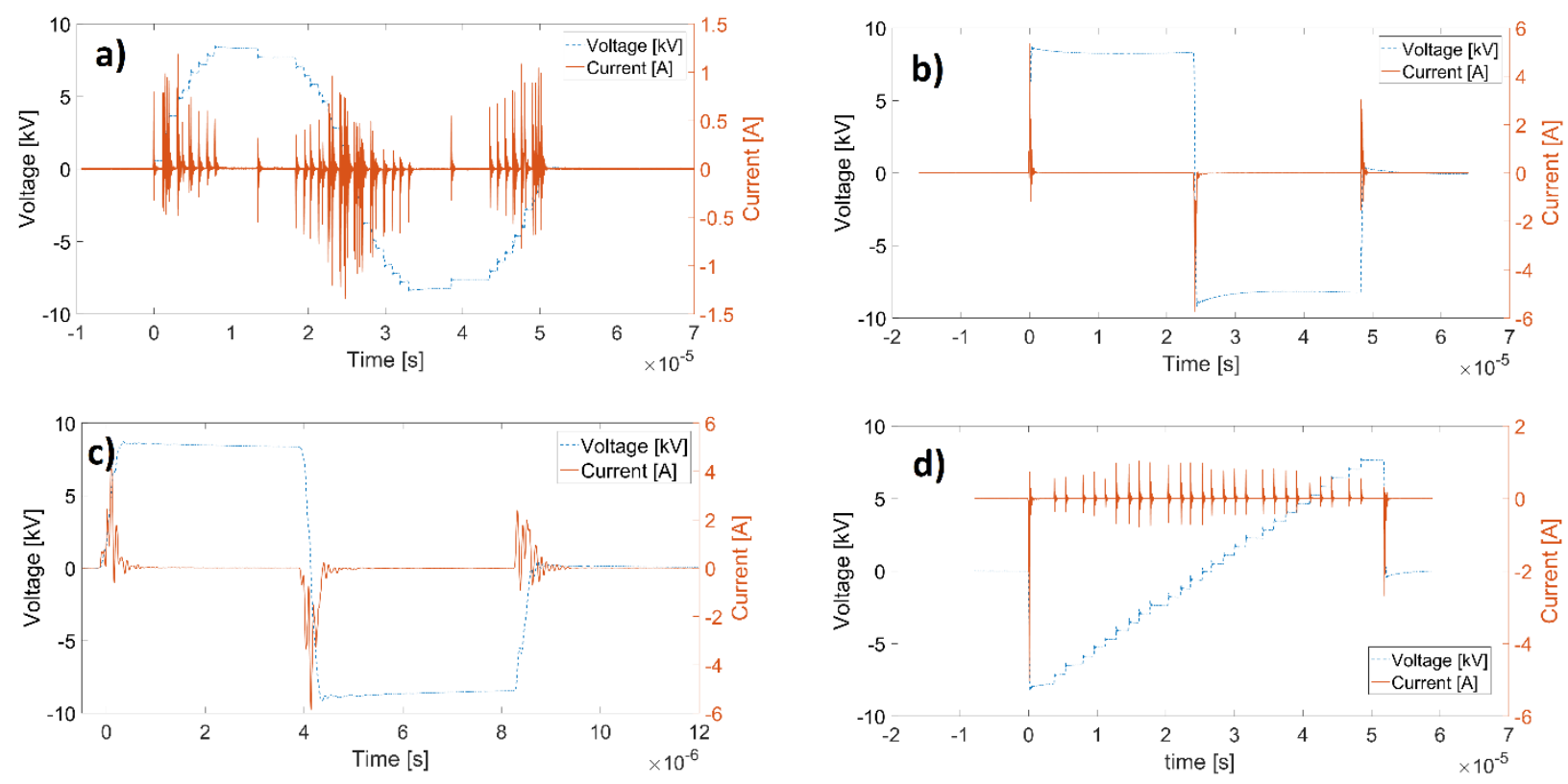

Figure 2 - Voltage (blue) and current (red) time behavior for (a) sinusoidal; (b) square; (c) pulse; and (d) sawtooth positive waveforms.

All experiments were performed at constant peak voltage $\left(8.4 \pm 0.2 \mathrm{kV}_{\mathrm{p}}\right)$ and average power $(16 \pm 1 \mathrm{~W})$, so a fair comparison about the efficacy of each waveform could be performed. A Tektronix P6015A capacitive compensated high-voltage probe with a bandwidth of $75 \mathrm{MHz}$ and a Tektronix TCP312 Hall current probe with a bandwidth of $100 \mathrm{MHz}$ were used to measure the applied voltage $v(t)$ and discharge current $i(t)$, respectively. Voltage and current signals were compensated for delays introduced by the probes and the average power was calculated by averaging the instantaneous power $p(t)=v(t) \cdot i(t)$ over one waveform period [20]. Power measurements were also compared with those obtained using the Lissajous method [21] and

11 both methods agreed with each other within a $10 \%$.

All waveforms except for the pulse had a frequency of $20 \mathrm{kHz}$ (period $50 \mu \mathrm{s}$ ). The pulse waveform, as a matter of fact, is a square waveform with a frequency of $125 \mathrm{kHz}$ (period $8 \mu \mathrm{s}$ ). This is the highest frequency that can be generated with the supply source used in this work and has been chosen to test the ozone production efficiency when the rising and falling edges of the voltage waveform are temporally close to each other. In order to maintain constant frequency, peak voltage and average power for all waveforms, the applied voltage was modulated and the duty-cycle (equation 1) was adjusted accordingly. In particular, the duty-cycle was set to $30 \%$ for the sinusoidal, $27 \%$ for the sawtooth, $18 \%$ for the square and $4 \%$ for the pulse waveform. 
2 In order to clarify different duty cycle percentages for the abovementioned waveforms, a sketch of one 3 applied voltage waveforms for each duty cycle period is reported in Figure 3.

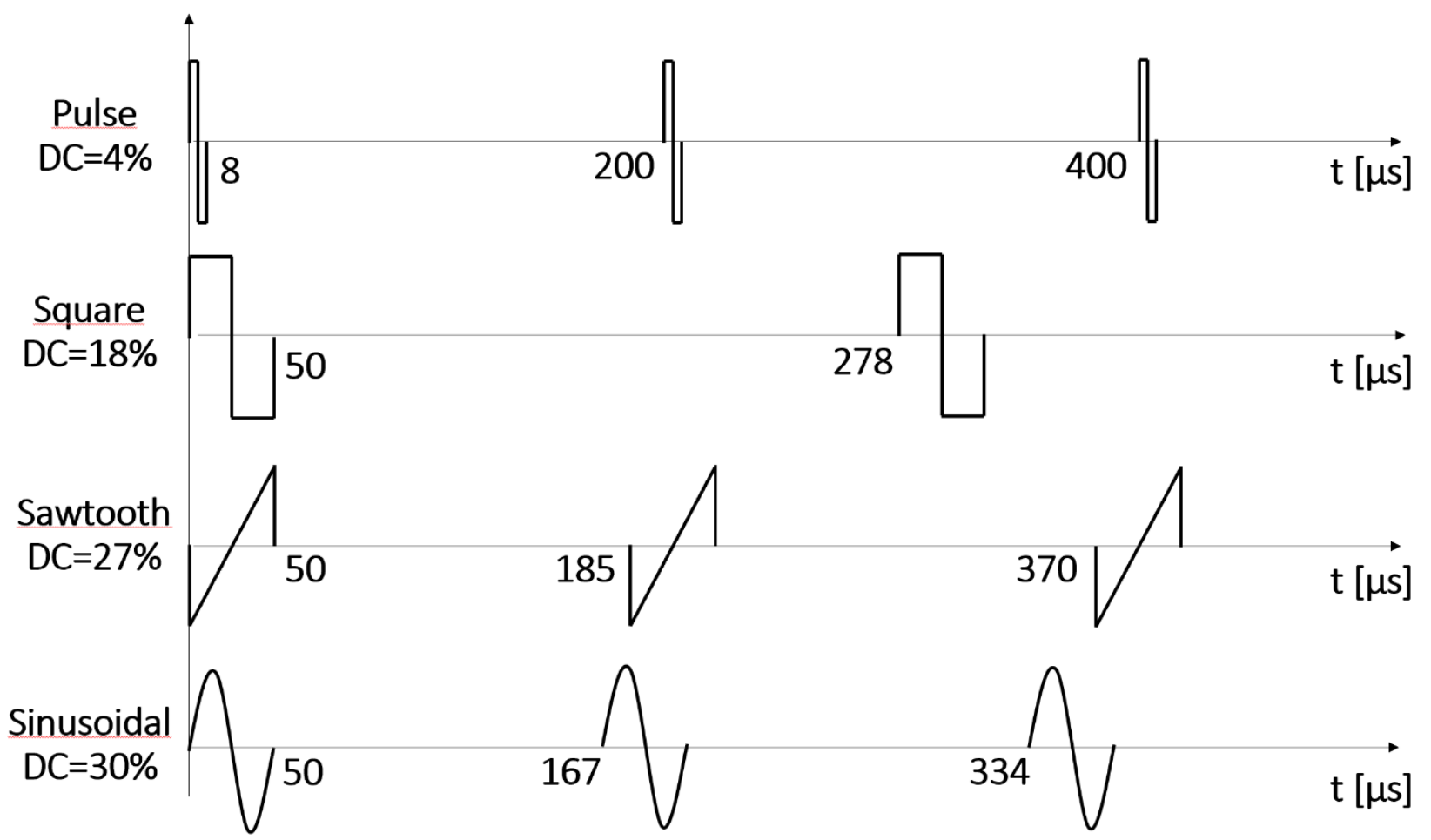

Figure 3 - Applied voltage waveforms with 1 period during the on-time. For each waveform, the duty cycle (DC) is adjusted so that the average power and peak voltage are the same in all cases.

Due to the topology of the multilevel power supply, the applied voltage does not suffer from switching-on and switching-off transients often encountered in power supplies with other topologies (e.g. resonant power supplies). Therefore, it is possible to drive the plasma even with just 1 period of the desired waveform (as shown in figure 2). Besides the change in applied voltage waveform duty cycle, we have also investigated the effect of the on-time on the ozone production efficacy. On times corresponding to 1, 10, 100 and 1000 waveforms periods in each duty cycle time interval have been chosen.

\subsection{Plasma-liquid micro-bubble reactor}

A dielectric barrier discharge (DBD) micro-bubble reactor was used in this study. The device produces plasma beneath the liquid and in the immediate proximity of the gas-liquid interface where bubbles form. This is possible by using a woven stainless-steel membrane (Plastok, UK) as both ground electrode and site 
1 of bubble generation. The membrane has a diameter of $8 \mathrm{~cm}$ an aperture of $35 \mu \mathrm{m}$ and a wire diameter of 30

$2 \mu \mathrm{m}$. At a gap of $1 \mathrm{~mm}$ below the membrane are the tips of the 19 high voltage electrodes. These consist of

3 stainless-steel rods with a hemispherical cap at the tip, placed within a quartz crucible with a wall thickness

4 of $1.5 \mathrm{~mm}$. This array of high voltage electrodes is held within a PTFE mount where the compressed air is

5 supplied tangentially at 1 standard liter per minute (SLPM) using a mass flow controller (MKS, PR4000B).

6 Holes in the top of the PTFE mount allow gas to flow towards the plasma discharge before forming the

7 bubbles in the tank above. The tank has a volume of $1.1 \mathrm{~L}$ and at its center there is a draft tube, which as

8 bubbles rise induces convection currents, effectively mixing the solution and eliminating the need for

9 mechanical mixing [22]. Although quartz crucibles and a stainless steel membrane were used in this study,

10 the rest of reactor is the same as that reported in [23,24].

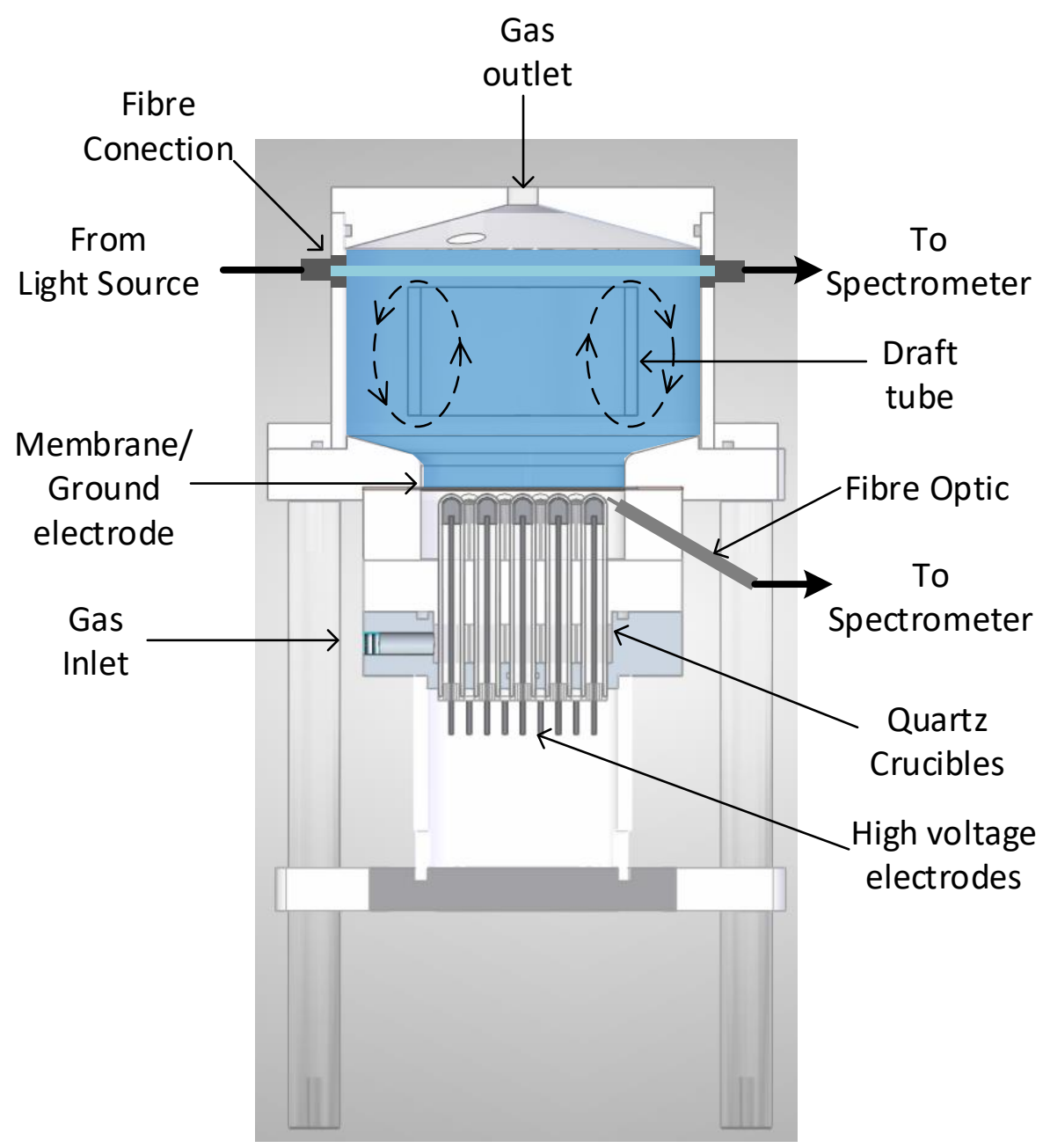


2 To measure the ozone in the deionized water the UV-absorption was used [25,26]. The ozone transferred

3 from the plasma to the deionized water was quantified using the Beer-Lambert law. Figure 4 (a) shows a

4 schematic of the reactor indicating how an ultraviolet (UV) LED source (LLS-255, Ocean Optics) was

5 directed through the liquid using a collimating lens and optical fibers. A bubble deflector was used to avoid

6 bubble interference in the ozone measurements. A second lens focuses the light onto an optical fibre. The

7 intensity of the collected light is measured with a spectrometer (HR2000+, Ocean Optics). By measuring the transmitted light intensity at $254 \mathrm{~nm}$ and knowing the optical path length of the system $(14 \mathrm{~cm})$, the ozone concentration can be determined. A molar absorption coefficient of $3300 \mathrm{M}^{-1} \mathrm{~cm}^{-1}$ was used calculated by in the calculations [27]. Since changes induced in the temperature of the liquid temperature were less than 3 degrees and the optical cross section of ozone molecules at $254 \mathrm{~nm}$ is only weakly dependent on temperature [28], temperature effects were neglected.

\section{$2.4 \mathrm{pH}$ and Conductivity}

14 Changes in $\mathrm{pH}$ and electrical conductivity in the treated water were monitored by taking $15 \mathrm{~mL}$ samples before and after the plasma treatment. $\mathrm{pH}$ and electrical conductivity values were measured using a benchtop meter (A215, Thermo Scientific).

\subsection{Indigo Probe}

The combined effect of reactive oxidative species and UV light on the liquid was assessed with Potassium Indigo Trisulfonate (Sigma Aldrich). This chemical probe has a high molar absorptivity $\left(23,800 \mathrm{M}^{-1} \mathrm{~cm}^{-1}\right)$ at $605 \mathrm{~nm}$ [29], and it is readily oxidized by reactive oxygen species (e.g. ozone, hydrogen peroxide, hydroxy radicals) and UV light produced in the plasma. A $1.1 \mathrm{~L}(0.1 \mathrm{M})$ solution of Potassium Indigo Trisulfonate was prepared with in-situ concentrations measured using the same experimental set up used for the ozone measurements, with the substitution of the UV LED for a white halogen light source (HL-2000, Ocean Optics). Although proposed as a standard method to measure ozone concentration due to its high sensitivity [30], its lack of selectivity can lead to indigo degradation trends being different to those obtained measured by UV absorption when there are multiple sources of oxidation, such as from a plasma. 


\subsection{Optical Emission Spectroscopy}

2 The optical emission of the plasma was measured using an optic fibre through a sealed hole in the plasma

3 module as shown in Figure 4a. The fibre terminal was connected to an Andor SR-303i-A Czerny-Turner

4 spectrograph with an entrance slit of $10 \mu \mathrm{m}$ and a holographic grating of $24001 \mathrm{~mm}^{-1}$. The detector was a

5 CCD camera paired to the spectrograph and set to an exposure time of $100 \mathrm{~ms}$. A total amount of 1000

6 acquisitions were acquired per measurement. Calibration of the spectra in intensity and wavelength was

7 carried out with a Deuterium- Halogen light source (DH-2000-Bal, Ocean optics) and a Mercury-Argon lamp

8 (HG-1, Ocean Optics), respectively. The plasma gas temperature was estimated by measuring the rotational

9 temperature of the 0-2 and 0-3 ro-vibrational bands of the nitrogen $C^{3} \Pi_{u}-B^{3} \Pi_{g}$ second positive system

10 transition and comparing them to a synthetic spectra generated with SPECAIR $[31,32]$.

\subsection{Ozone diffusion calculation}

12 Ozone diffusion out of a discharge channel in the gas phase was estimated by solving the time dependent 13 diffusion equation (2):

$$
\frac{\partial C_{O_{3}}}{\partial t}+\nabla \cdot\left(-D_{O_{3}} \nabla C_{O_{3}}\right)=o
$$

Plasma streamers are usually characterized by diameters in the order of 10-100 $\mu \mathrm{m}$ [33] and for this study we assumed a typical diameter of $60 \mu \mathrm{m}$. A filamentary discharge was modelled as a cylinder and the ozone diffusion coefficient used in the calculation was $0.5 \mathrm{~cm}^{2} \mathrm{~s}^{-1}$ [34-36]. The initial conditions assume a

18 cylindrical channel containing ozone and the effect background gas flow is not modelled as this is expected 19 to be negligible for the time scale and dimensions relevant to the calculations (microseconds and micrometers). The simulation was performed a Neumann boundary condition away from the discharge

21 channel):

$$
-D_{O_{3}} \nabla C_{O_{3}}=0
$$




\section{$1 \quad 3.0$ Results and Discussion}

2 The results of the study are presented in the following two subsections. The first one (section 3.1) considers

3 the effect of the waveform on the production of ozone and the degradation of indigo dye in the liquid; and

4 the second one (section 3.2) the effect of the plasma on-time.

\section{$5 \quad 3.1$ Effect of waveform}

6 To assess the effect of the voltage waveform shape on the ozone production efficiency of the device, the 7 applied voltage was modulated so that one complete period of the desired waveform was applied during the 8 plasma on-time (50 $\mu$ s for the sinusoidal, square and sawtooth waveforms; $8 \mu$ s for the pulse waveform; see 9 Figure 2). The peak voltage $\left(8.4 \pm 0.2 \mathrm{kV}_{\mathrm{p}}\right)$ and the average power $(16 \pm 1 \mathrm{~W})$ were kept the same for all the 10 experiments and this was achieved by adjusting the off-time (i.e. duty-cycle) as necessary (as explained in 11 Section 2.1).

12 Figure 5a shows the time evolution of ozone concentration in the liquid. The square waveform results in the 13 production of the largest amount of dissolved ozone, outperforming the sine wave, which in turn produces 14 slightly higher concentrations than the sawtooth and pulse waveforms. The higher concentration of ozone generated by the square wave also translates in a faster degradation of the indigo dye (Figure 5b). However, the trends of ozone and indigo are not identical due to the non-selective nature of indigo. Indigo can be oxidized by many ROS, including hydroxyl radicals [37] and superoxide [38], which are likely to be formed 18 at different concentrations from each waveform. As expected, the air plasma treatments resulted in acidification of the liquid and an increase in its conductivity. This is caused by the generation of reactive nitrogen species (RNS), such as $\mathrm{NO}, \mathrm{N}_{2} \mathrm{O}, \mathrm{NO}_{2}$ that

21 lead to the production of nitrous and nitric acid [39]. Interestingly, the lowest changes in $\mathrm{pH}$ and conductivity were obtained with the square waveforms (Figure $5 \mathrm{c}$ and d), which are the ones that resulted in highest ozone concentrations. This therefore points towards waveforms favoring the production of either ozone, such as the square waveform or RNS, such as the pulse and sawtooth waveforms. Increasing the gas temperature is a 
1 by $\mathrm{NO}$ and $\mathrm{NO}_{2}$ to form $\mathrm{NO}_{2}$ and $\mathrm{NO}_{3}$ respectively double when the temperature increases from $300 \mathrm{~K}$ to $2320 \mathrm{~K}[39]$.
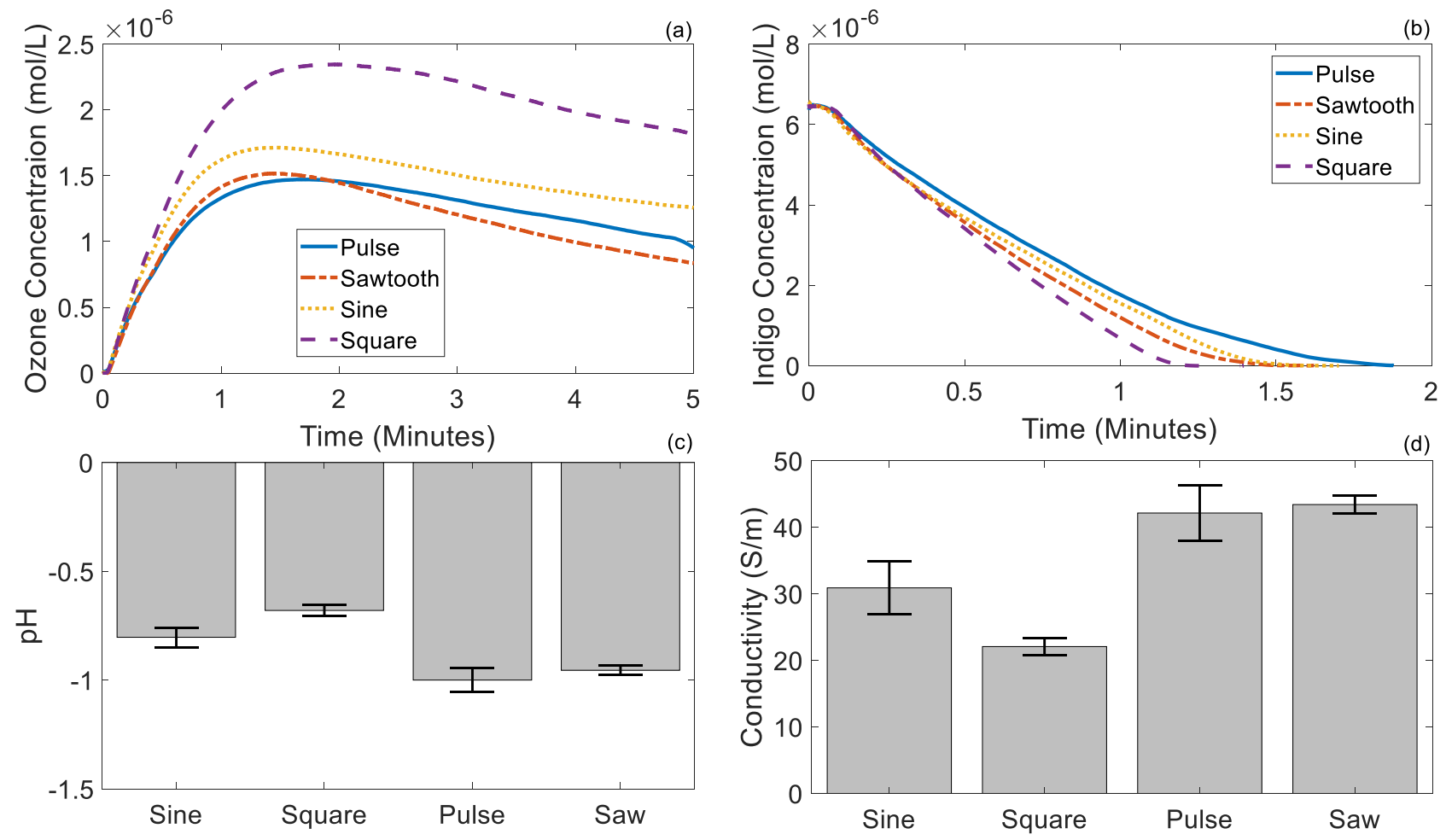

Figure 5 - The effects of each waveform in the liquid observing the change in (a) ozone and (b) indigo concentration plus the change in (c) $\mathrm{pH}$ and (d) conductivity.

To determine whether the temperature was the factor in the system favoring RNS production, the average temperature of the discharge was estimated by measuring the rotational temperature of the second positive system of nitrogen molecules. Figure 6 shows the emission spectra of the 0-2 and 0-3 ro-vibrational transition of the $\mathrm{N}_{2}$ SPS for plasmas sustained with different waveforms and the SPECAIR's synthetic 11 spectra fits. All plasmas have an average rotational temperature of $T_{\text {rot }}=380 \pm 15 \mathrm{~K}$. This result differs from those reported in [19] where different waveforms resulted in different rotational temperatures. However, it is important to notice that in that study different waveforms deposited different amount of power in the discharge, whilst in this study the average power was kept constant for all waveforms. 

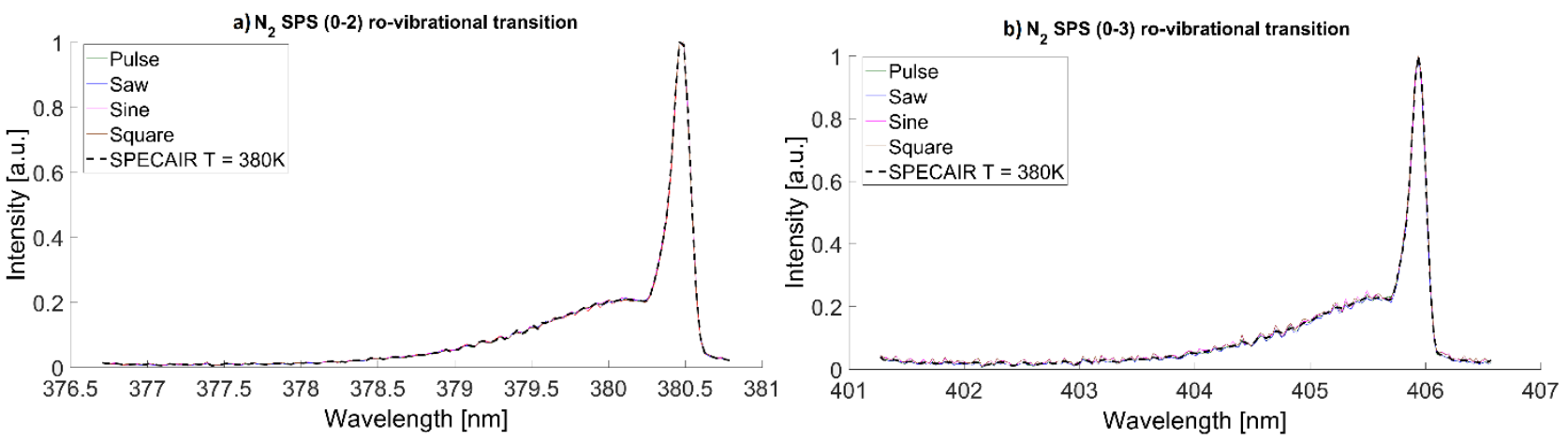

Figure 6 - Optical emission spectra for all four waveforms and their fit to SPECAIR's synthetic spectrum. (a) 0-2 and (b) 0-3 ro-vibrational bands.

Although the average power and the average gas plasma temperature are the same in all cases, different waveforms deliver the energy in different ways to the discharge. Waveforms characterized by fast voltage variations, i.e. large $\mathrm{dV} / \mathrm{dt}$ values, are more effective in creating strong electric fields in the discharge gap [6]. This generates higher energy electrons and stronger avalanches during gas breakdown, which subsequently create plasmas with a higher degree of dissociation and ionization. Consequently, when the square waveform is applied, an elevated number of energetic electrons are available to trigger and enhance chemical reaction pathways, such as the dissociation of molecular oxygen required for ozone production. This behavior explains why the square waveform outperforms the sinusoidal and sawtooth waveforms (Figure 5a). However, it is important to notice that the square and pulse waveforms have the same dV/dt values, indicating that other factors are equally important in determining the final efficacy of the applied voltage waveform. A possible explanation can be found in the ozone diffusion out of the discharge channel after each discharge. This behavior is discussed below.

Since the square and the pulse waveforms have the same rising and falling fronts (see Figure 2), the difference in their performance must be related to the time interval between these fronts. More precisely, we argue that the difference in performance is related to the ability of the ozone produced in the rising edge of the pulse to diffuse away from the discharge region before the arrival of the falling edge. For a mass flow rate of 1 SLPM, a membrane diameter of $8 \mathrm{~cm}$ and a discharge gap of $1 \mathrm{~mm}$, the gas residence time within the discharge gap is $\sim 300 \mathrm{~ms}$. This time is about 4 orders of magnitude larger than the separation between rising and falling edges of the pulse and square waveforms $(8-50 \mu \mathrm{s})$. Therefore, in a first order approximation, it can be assumed that each plasma avalanche is ignited in a stationary background gas. The 
1 discharges for all waveforms described in this study are filamentary and ozone is produced within each

2 plasma filament and then diffuses into the surrounding gas [33]. Figure 7 shows the diffusion of ozone after

3 its formation within a plasma filament according to the calculation described in section 2.7 and referring to

4 the waveform train reported in Figure 3. For convenience, the normalized ozone concentration at $t=0$ is assumed to be uniform within the filament (Figure 7a). As time progresses, the ozone diffuses away. Figure $7 \mathrm{~b}$ shows ozone distribution after $4 \mu$ s and Figure $7 \mathrm{c}$ after $25 \mu$ s. Figure $7 \mathrm{~d}$ shows the time evolution of the ozone concentration at the center of the plasma filament as a function of time.
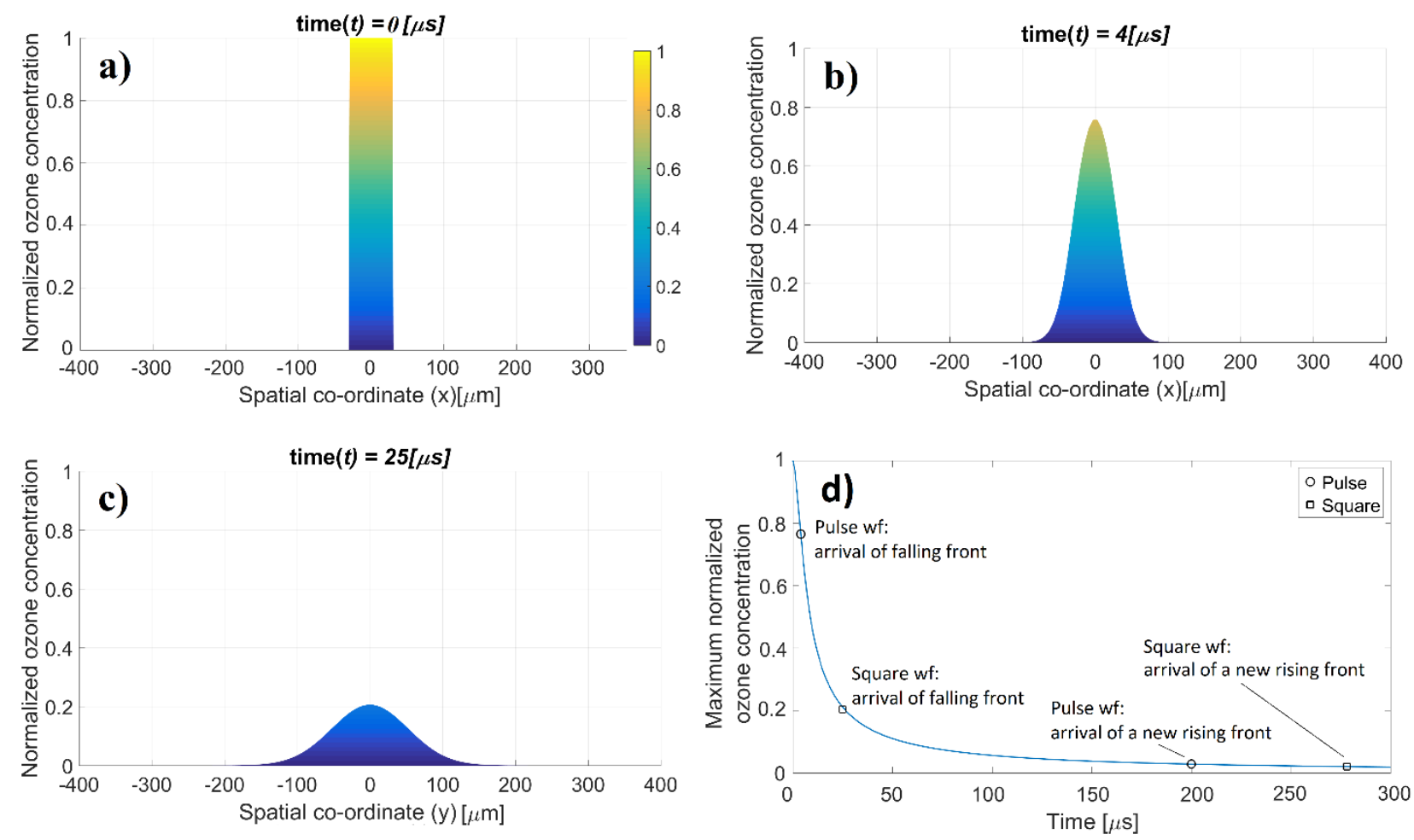

Figure 7 - Normalized ozone profiles at (a) $t=0 \mu$ s (b) $4 \mu$ s and (c) $25 \mu$ s after a filamentary discharge. (d) Time evolution of the normalized ozone concentration at the location of the discharge.

11 In an ideal situation, the ozone generated in one plasma avalanche should be transported away from the discharge region before the arrival of a new discharge, as the ignition of a new discharge will destroy some of the background ozone present in the gas. For the square waveform, the arrival of the falling front happens $25 \mu$ s after the rising front (see Figure 3 and Figure 7c, d). At that time, the ozone created by the rising edge discharge has diffused away and only $20 \%$ of the original ozone remains within the discharge channel. On the contrary, for the pulse waveform, the falling front arrives only $4 \mu$ s after the rising edge (see Figure 3 and Figure $7 \mathrm{~b}, \mathrm{~d})$. This means that more than $75 \%$ of the ozone created by the rising front still remains in the 
1 discharge channel and will therefore be partially destroyed by the discharge created by the falling edge of the

2 voltage waveform. This destruction of ozone can be inferred from the enhanced formation of RNS and

3 increased acidification seen in Figure 5 c) and d) for the pulse discharge. It is therefore concluded that the

4 longer time between the rising and falling fronts in the square waveform allows a more efficient formation of

5 ozone. When the second waveform train is produced (Figure 7d), residual ozone concentration is below the

$65 \%$ for both waveforms. This reasoning can also be applied to understand the similarity in ozone trends

7 between the sawtooth and the pulse waveforms. As the sawtooth waveform contains 28 voltage wave fronts

8 during the on-time period, the time interval between each front is about $2 \mu \mathrm{s}$. As far as this value is quite

9 close to that of the pulse waveform $(4 \mu \mathrm{s})$, the ozone is partially destroyed shortly after its production also

10 with the sawtooth voltage shape.

\subsection{Effect of on time}

12 To assess the effect of the on-time on the performance of the device, the applied voltage was modulated with 13 on-times of $0.05,0.5,5$ and $50 \mathrm{~ms}$, which correspond to $1,10,100$ and 1000 periods of a $20 \mathrm{kHz}$ signal.

14 Notwithstanding that the square waveform is more efficient than the sinusoidal waveform, we explore the

15 effect of the on-time on the performance of the device for sinusoidal waveforms because most DBDs are 16 driven by sinusoidal voltages. The peak voltage $\left(8.4 \pm 0.2 \mathrm{kV}_{\mathrm{p}}\right)$, the average power $(16 \pm 1 \mathrm{~W})$ and the duty17 cycle (30\%) were kept the same for all the experiments.

Figure 8a shows the time evolution of the ozone concentration in the liquid for different on-times. Shorter on-times result in larger ozone concentrations and this translates in a faster degradation of indigo dye (Figure $8 b)$. The improved performance for shorter on-times can be attributed to the fact that ozone generated in an

21 'on' period has time to diffuse away before the next discharge is generated, as already discussed in the previous section. As expected, the air plasma treatments result in acidification of the liquid and increased 23 conductivity, although no significant difference between the various on-times was observed. These results 24 suggest that shorter modulations have little effect on the RNS chemistry but do improve the ozone production. 

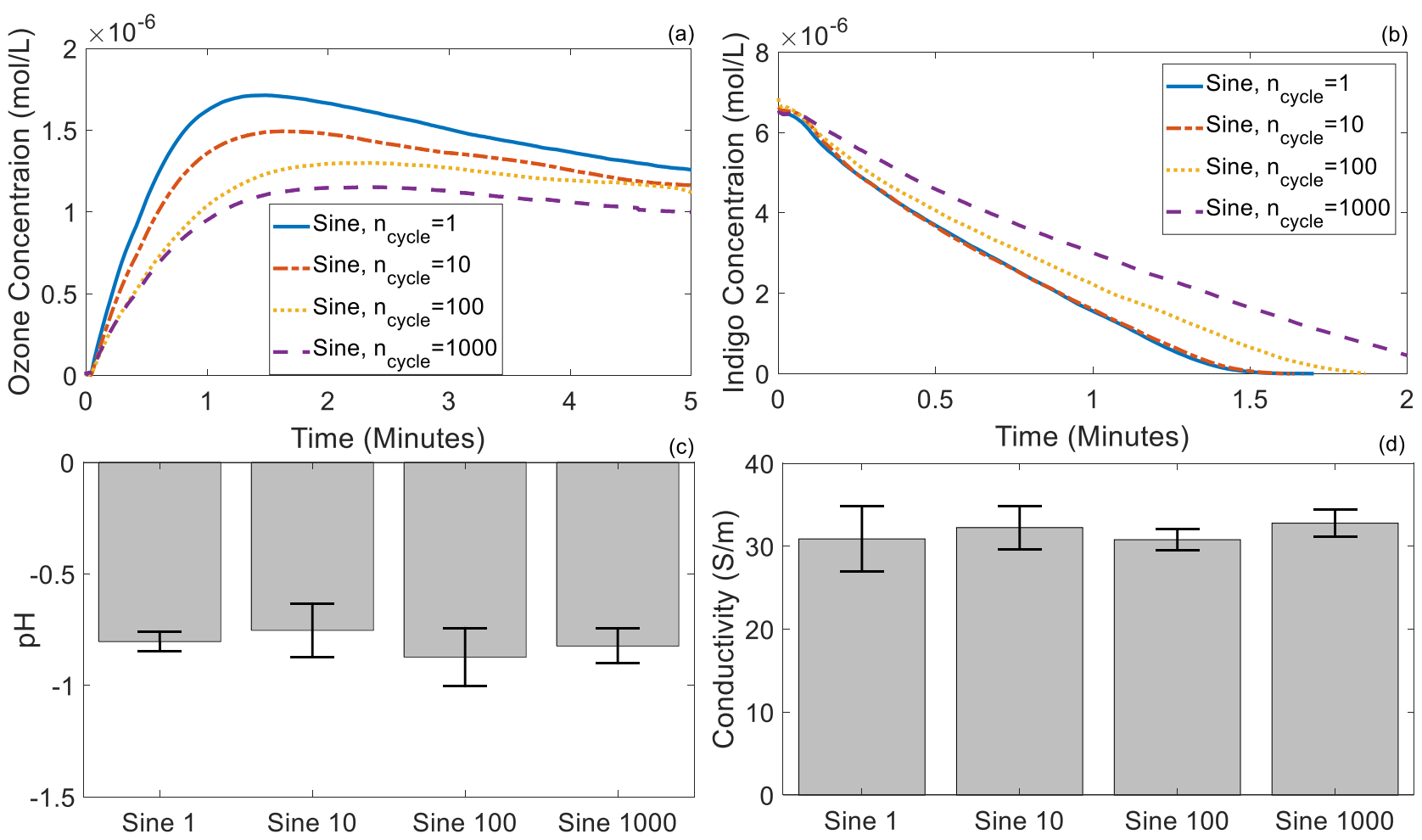

Figure 8 - The effects of the modulation number on the liquid observing the change in (a) ozone and (b) indigo concentration plus the change in (c) $\mathrm{pH}$ and (d) conductivity.

\subsection{Conclusion}

Dielectric barrier discharges have received renewed attention in recent years for their potential use in biomedical, agricultural and environmental applications. While a large number of publications demonstrate their potential in these fields, little attention has been paid to the efficacy of the devices and ways to optimize it.

In this study we have analyzed the ozone production efficiency of a DBD micro-bubble reactor when the device is operated at constant input power but with different shape and on-time duration of the applied voltage waveform. This was possible by using a custom-built multilevel power supply capable of producing arbitrary voltage waveforms without the switching-on and switching-off transients often encountered in power supplies with other topologies.

Although temperature is a well-known factor in determining the efficacy of ozone generators, performance differences observed when different waveforms and on-times are used could not be attributed to temperature 
1 variations in this study. Instead, it was found that the efficacy of the different waveforms depends strongly

2 on the time in between subsequent discharges as better efficacy is obtained when ozone generated in an

3 avalanche has enough time to diffuse away from the discharge region before a new avalanche forms. This

4 guarantees that ozone produced in a discharge event is not destroyed in a subsequent one and it also limits

5 the poisoning by reactive nitrogen species of the background gas. As a result, square waveforms are found to

6 be more effective than sinusoidal, sawtooth and short pulse waveforms and for a fixed duty cycle, shorter on-

$7 \quad$ time periods are preferred over longer ones.

$8 \quad$ References

9 [1] Glaze W H 1987 Drinking-water treatment with ozone Environ. Sci. Technol. 21 224-30

10 [2] Langlain B, Reckhow D and Brink D 1991 Ozone in Water Treatment: Application and Engineering

11 [3] Vezzu G, Lopez J L, Freilich A and Becker K H 2009 Optimization of large-scale ozone generators IEEE Trans. Plasma Sci. 37 890-6

13 [4] Murata T, Okita Y, Noguchi M and Takase I 2004 Basic parameters of coplanar discharge ozone generator Ozone Sci. Eng. 26 429-42

15 [5] Brueggemann N, Puehmeier T, Fiekens R, Richardt F J and Salvermoser M 2017 Cooling Conditions of Ozone Generators Ozone Sci. Eng. 39 196-201

[6] Sung T L, Teii S, Liu C M, Hsiao R C, Chen P C, Wu Y H, Yang C K, Teii K, Ono S and Ebihara K

[7] Zhang X, Lee B J, Im H G and Cha M S 2016 Ozone Production With Dielectric Barrier Discharge: Effects of Power Source and Humidity IEEE Trans. Plasma Sci. 44 2288-96

[8] Facta M, bin Salam Z and Bin Buntat Z 2009 The development of ozone generation with low power consumption Innov. Technol. Intell. Syst. Ind. Appl. 2009. CITISIA 2009 440-5 

frequency and ozone generation for surface dielectric barrier discharges in atmospheric air Sci. Rep. 7 $1-11$

[10] Haverkamp R G, Miller B B and Free K W 2002 Ozone Production in a High Frequency Dielectric Barrier Discharge Generator Ozone Sci. Eng. 24 321-8

[11] Suksri A, Karnchanalekha K, Tonmitra K and Apiratikul P 2009 A comparative study on suitable high voltage sources for ozone generation 2009 6th Int. Conf. Electr. Eng. Comput. Telecommun. Inf. Technol. ECTI-CON $20091296-9$

[12] Šimek M, Pekárek S and Prukner V 2010 Influence of power modulation on ozone production using an AC surface dielectric barrier discharge in oxygen Plasma Chem. Plasma Process. 30 607-17

[13] Ma T, Jiang H, Liu J and Zhong F 2016 Decomposition of Benzene Using a Pulse-Modulated DBD Plasma Plasma Chem. Plasma Process. 36 1533-43

[14] Olszewski P, Li J F, Liu D X and Walsh J L 2014 Optimizing the electrical excitation of an atmospheric pressure plasma advanced oxidation process J. Hazard. Mater. 279 60-6

[15] Barni R, Biganzoli I, Dell'Orto E C and Riccardi C 2015 Effect of duty-cycles on the air plasma gasphase of dielectric barrier discharges J. Appl. Phys. 118

[16] Abdelaziz A A, Seto T, Abdel-Salam M, Ishijima T and Otani Y 2015 Influence of applied voltage waveforms on the performance of surface dielectric barrier discharge reactor for decomposition of naphthalene J. Phys. D. Appl. Phys. 48 1-9

[17] Mericam-Bourdet N, Kirkpatrick M J, Tuvache F, Frochot D and Odic E 2012 Effect of voltage waveform on dielectric barrier discharge ozone production efficiency Eur. Phys. J. Appl. Phys. 57 30801

[18] Dragonas F A, Neretti G, Sanjeevikumar P and Grandi G 2015 High-Voltage High-Frequency Arbitrary Waveform Multilevel Generator for DBD Plasma Actuators IEEE Trans. Ind. Appl. 51 $3334-42$ 
1 [19] Borghi C A, Cristofolini A, Grandi G, Neretti G and Seri P 2015 A plasma aerodynamic actuator supplied by a multilevel generator operating with different voltage waveforms Plasma Sources Sci.

\section{Technol. 24}

[20] Neretti G, Taglioli M, Colonna G and Borghi C A 2017 Characterization of a dielectric barrier discharge in contact with liquid and producing a plasma activated water Plasma Sources Sci. Technol. 26

[21] Manley T C 1943 The Electric Characteristics of the Ozonator Discharge Trans. Electrochem. Soc. 8483

[22] Ying K, Gilmour D J, Shi Y and Zimmerman W B 2013 Growth Enhancement of Dunaliella salina by Microbubble Induced Airlift Loop Bioreactor (ALB)—-The Relation between Mass Transfer and Growth Rate J. Biomater. Nanobiotechnol. 04 1-9

[23] Wright A, Bandulasena H, Ibenegbu C, Leak D, Holmes T, Zimmerman W, Shaw A and Iza F 2018 Dielectric barrier discharge plasma microbubble reactor for pretreatment of lignocellulosic biomass AIChE J. $643803-16$

[24] Wright A, Marsh A, Ricciotti F, Shaw A, Iza F, Holdich R and Bandulasena H 2018 Microbubbleenhanced dielectric barrier discharge pretreatment of microcrystalline cellulose Biomass and Bioenergy 118 46-54

[25] Van den Broeke J, Ross P S, van der Helm A W C, Baars E T and Rietveld L C 2008 Use of on-line UV/Vis-spectrometry in the measurement of dissolved ozone and AOC concentrations in drinking water treatment Water Sci. Technol. 57 1169-75

[26] Majewski J 2012 Methods for measuring ozone concentration in ozone-treated water Prz. Elextrotechniczny 253-5

[27] Hart E J, Sehested K and Holcman J 1983 Molar Absorptivities of Ultraviolet and Visible Bands of Ozone in Aqueous Solutions Anal. Chem. 55 46-9

[28] Grebenshchikov S Y, Qu Z-W, Zhu H and Schinke R 2007 New theoretical investigations of the 
photodissociation of ozone in the Hartley, Huggins, Chappuis, and Wulf bands. Phys. Chem. Chem. Phys. 9 2044-64

[29] Bader H and Hoigné J 1981 Determination of ozone in water by the indigo method Water Res. 15 $449-56$

[30] Eaton A D, Clesceri L S, Greenberg A E and Franson M A H 1998 Standard methods for the examination of water and wastewater (Washington, DC: American Public Health Association)

[31] Laux C O 2002 Physico-Chemical Modeling of High Enthalpy and Plasma Flows (Rhode-SaintGenese, Belgium)

[32] Taglioli M, Shaw A, Wright A, FitzPatrick B, Neretti G, Seri P, Borghi C a and Iza F 2016 EHDdriven mass transport enhancement in surface dielectric barrier discharges Plasma Sources Sci. Technol. 25 06LT01

[33] Kogelschatz U 2002 Filamentary, patterned, and diffuse barrier discharges IEEE Trans. Plasma Sci. $301400-8$

[34] Hegeler F and Akiyama H 1997 Spatial and temporal distributions of ozone after a wire-to-plate streamer discharge IEEE Trans. Plasma Sci. 25 1158-65

[35] Faghri A, Zhang Y and Howell J 2010 Advanced Heat and Mass Transfer (Columbia: Global Digital Press)

[36] Neretti G, Taglioli M and Borghi C A 2018 Experimental determination and numerical evaluation under simplifying assumptions of the ozone concentration in an atmospheric-pressure air DBD plasma Eur. Phys. J. D 72113

[37] Wentworth P 2002 Evidence for Antibody-Catalyzed Ozone Formation in Bacterial Killing and Inflammation Science (80-. ). $2982195-9$

[38] Kettle A J, Clark B M and Winterbourn C C 2004 Superoxide converts indigo carmine to isatin sulfonic acid: Implications for the hypothesis that neutrophils produce ozone J. Biol. Chem. 279 
2 [39] Sakiyama Y, Graves D B, Chang H-W, Shimizu T and Morfill G E 2012 Plasma chemistry model of 3 surface microdischarge in humid air and dynamics of reactive neutral species J. Phys. D. Appl. Phys.

4 45425201

5 